\title{
Operators on a Hilbert space similar to a part of the backward shift of multiplicity one
}

\author{
by \\ Yoichi Uetake (Poznań)
}

\begin{abstract}
Let $A: X \rightarrow X$ be a bounded operator on a separable complex Hilbert space $X$ with an inner product $\langle\cdot, \cdot\rangle_{X}$. For $b, c \in X$, a weak resolvent of $A$ is the complex function of the form $\left\langle(I-z A)^{-1} b, c\right\rangle_{X}$. We will discuss an equivalent condition, in terms of weak resolvents, for $A$ to be similar to a restriction of the backward shift of multiplicity 1 .
\end{abstract}

1. Introduction. We characterize an operator on a separable complex Hilbert space which is similar to a part of the backward shift of multiplicity 1 , in terms of cyclic vectors and weak resolvents belonging to the Hardy space $\mathbb{H}^{\infty}$. We base our development on the work of Fuhrmann [6], Helton [7], Nikol'skiu [10], and Radjavi and Rosenthal [12]. We hope that this note would serve to unify and refine some results in the previous works mentioned above. What plays the central role is the concept of weak resolvent introduced by Fong, Nordgren, Radjavi and Rosenthal [5], [11], [13]. The weak resolvents here correspond to a class of characteristic functions of the Sz.-Nagy-Foias operator model theory [16]. Note that this operator model theory has been related to the Lax-Phillips scattering theory by Adamjan and Arov [1]. See also Helton [7] and Uetake [18].

For $A: X \rightarrow X$ a bounded operator on a complex Hilbert space $X$ with inner product $\langle\cdot, \cdot\rangle_{X}$ and $b, c \in X$, we define a weak resolvent of $A$ to be

$$
\left\langle(I-z A)^{-1} b, c\right\rangle_{X} .
$$

Here $I$ denotes the identity map on $X$. For this see also Mlak [9] and Uetake [17], [18].

Let $\mathbb{T}=\{z:|z|=1\}$, the unit circle in the complex plane, and $\mathbb{D}=\{z$ : $|z|<1\}$, the open unit disc. We use the following two Hardy spaces:

2000 Mathematics Subject Classification: 47A10, 47A11. 


$$
\begin{aligned}
\mathbb{H}^{2}=\left\{f: f(z)=\sum_{n=0}^{\infty} f_{n} z^{n}, z \in \mathbb{D}\right. & \\
\|f\|_{2} & \left.=\lim _{r \rightarrow 1}\left\{\frac{1}{2 \pi} \int_{0}^{2 \pi}\left|f\left(r e^{i \theta}\right)\right|^{2} d \theta\right\}^{1 / 2}<\infty\right\}, \\
\mathbb{H}^{\infty}=\left\{f: f(z)=\sum_{n=0}^{\infty} f_{n} z^{n}, z \in \mathbb{D},\right. & \left.\|f\|_{\infty}=\lim _{r \rightarrow 1} \max _{0 \leq \theta<2 \pi}\left|f\left(r e^{i \theta}\right)\right|<\infty\right\} \subset \mathbb{H}^{2} .
\end{aligned}
$$

Let $\mathbb{L}^{2}$ denote the Hilbert space of square integrable functions on $\mathbb{T}$, equipped with the inner product defined by

$$
\langle f, g\rangle=\frac{1}{2 \pi} \int_{0}^{2 \pi} f\left(e^{i \theta}\right) \overline{g\left(e^{i \theta}\right)} d \theta=\sum_{n=-\infty}^{\infty} f_{n} \bar{g}_{n}
$$

for $f(z)=\sum_{n=-\infty}^{\infty} f_{n} z^{n}, g(z)=\sum_{n=-\infty}^{\infty} g_{n} z^{n}, z \in \mathbb{T}$. Note that $\left\{z^{n}\right.$ : $z \in \mathbb{T}, n \in \mathbb{Z}\}$ is an orthonormal basis of $\mathbb{L}^{2}$. Then $\mathbb{H}^{2}$ is isometrically isomorphic to $\left\{f \in \mathbb{L}^{2}: f_{n}=0, n<0\right\} \subset \mathbb{L}^{2}$. Therefore we identify $\mathbb{H}^{2}$ with this subspace of $\mathbb{L}^{2}$ in the natural way and denote the inner product of $\mathbb{H}^{2}$ also by $\langle\cdot, \cdot\rangle$.

The unilateral shift of multiplicity 1 , denoted by $S: \mathbb{H}^{2} \rightarrow \mathbb{H}^{2}$, is defined by $(S f)(z)=z f(z)$, and its adjoint, the backward shift of multiplicity 1 , denoted by $S^{*}: \mathbb{H}^{2} \rightarrow \mathbb{H}^{2}$, is defined by

$$
\left(S^{*} f\right)(z)=\frac{f(z)-f(0)}{z}=\sum_{n=0}^{\infty} f_{n+1} z^{n} .
$$

A subspace $K \subseteq \mathbb{H}^{2}$ is said to be $S$-invariant (resp. $S^{*}$-invariant) if $S K \subseteq K$ (resp. $S^{*} K \subseteq K$ ). For an $S^{*}$-invariant subspace $K \subseteq \mathbb{H}^{2}, S^{*} \mid K: K \rightarrow K$ is called a part (or restriction) of the backward shift of multiplicity 1 .

To state the main theorem we need to define some notions of cyclicity. Let $T: H \rightarrow H$ be a bounded operator on a separable complex Hilbert space $H$. Let $E_{0}=\left\{\left(u_{n}\right)_{n=0}^{\infty}: u_{n}=0\right.$ for all $n \geq N$ for some $\left.N\right\} \subset l^{2}=l^{2}(0, \infty)$. Given $v \in H$, define $\mathcal{C}: E_{0} \rightarrow H$ by $\mathcal{C} u=\sum_{n=0}^{\infty} u_{n} T^{n} v$ for $u=\left(u_{n}\right)_{n=0}^{\infty}$ $\in E_{0}$. Note that the sum is actually finite. Let us call $\mathcal{C}$ the cyclicity operator for $v$ and $T$. For this operator, see also Helton [7] and Fuhrmann [6]. Note that $\mathcal{C}$ is not necessarily bounded.

Definition 1.1. $v$ is cyclic for $T$ if $\overline{\operatorname{span}}\left\{T^{n} v: n \geq 0\right\}=H$, or, equivalently, $\overline{\mathcal{C}\left(E_{0}\right)}=H$.

Here $\overline{\text { span }}$ denotes the closure of the linear span. 
Definition 1.2. $v$ is continuously cyclic for $T$ if $v$ is cyclic for $T$ and $\mathcal{C}$ is continuous (i.e. bounded).

Note that if $\mathcal{C}: E_{0} \rightarrow H$ is continuous, then $\mathcal{C}$ extends to a continuous operator from $l^{2}$ to $H$ in the natural way, since $E_{0}$ is dense in $l^{2}$. Therefore $\mathcal{C}: E_{0} \rightarrow H$ is continuous if and only if it has a continuous extension $\mathcal{C}: l^{2} \rightarrow H$. If $v$ is continuously cyclic for $T$, then for the extended $\mathcal{C}$, $\overline{\mathcal{C}\left(l^{2}\right)}=H$.

Definition 1.3. $v$ is strongly cyclic for $T$ if $v$ is continuously cyclic for $T$ and the extended $\mathcal{C}: l^{2} \rightarrow H$ is surjective.

2. The main theorem. We can now state our main theorem.

Theorem 2.1 (Main Theorem). Let $A: X \rightarrow X$ be a bounded operator on a separable complex Hilbert space equipped with an inner product $\langle\cdot, \cdot\rangle_{X}$. There exists an $S^{*}$-invariant closed subspace $K \subseteq \mathbb{H}^{2}$ such that $A: X \rightarrow X$ is similar to $S^{*} \mid K: K \rightarrow K$, i.e. $A=T^{-1}\left(S^{*} \mid K\right) T$ for some bounded bijection $T: X \rightarrow K$, if and only if there exist $b, c \in X$ such that $b$ is continuously cyclic for $A, c$ is strongly cyclic for $A^{*}$ and the weak resolvent $\varphi(z)=\left\langle(I-z A)^{-1} b, c\right\rangle_{X}$ is in $\mathbb{H}^{\infty}$.

The following corollary is an immediate consequence of the above theorem.

Corollary 2.2. Let $A$ be a bounded operator on a separable complex Hilbert space $X$ with an inner product $\langle\cdot, \cdot\rangle_{X}$. If there exist $b, c \in X$ such that $b$ is continuously cyclic for $A, c$ is strongly cyclic for $A^{*}$ and the weak resolvent $\varphi(z)=\left\langle(I-z A)^{-1} b, c\right\rangle_{X}$ is in $\mathbb{H}^{\infty}$, then $r(A) \leq 1$, where $r(A)$ denotes the spectral radius of $A$.

Proof. By Theorem 2.1, $A$ is similar to $S^{*} \mid K$, a part of the backward shift of multiplicity 1 for some $K \subseteq \mathbb{H}^{2}$. Since $\left\|S^{*} \mid K\right\| \leq 1$, the assertion readily follows.

The above theorem is an extension of the following theorem of Radjavi and Rosenthal to separable infinite-dimensional Hilbert spaces.

Theorem 2.3 (Radjavi and Rosenthal [12, Theorem 3.27, p. 54]). Let $A$ be an operator on a finite-dimensional complex Hilbert space. Then $A$ is similar to a part of $S^{*}$ of multiplicity 1 if and only if $A$ is cyclic (or, equivalently, the characteristic and minimal polynomials coincide) and $r(A)<1$.

Here $A$ is said to be cyclic if there exists a cyclic vector for $A$. One can use the following theorem to deduce Theorem 2.3 from Theorem 2.1.

Theorem 2.4 (Uetake [17, Theorem 2]). Let $A$ be a bounded operator on a separable complex Hilbert space $X$ with an inner product $\langle\cdot, \cdot\rangle_{X} \cdot$ Let 
$b \in X$ be cyclic for $A$ and $c \in X$ be cyclic for $A^{*}$. Suppose that $(\lambda I-A)^{-1}$ is meromorphic in an open neighborhood of $\lambda_{0}$. Then $\psi(\lambda)=\left\langle(\lambda I-A)^{-1} b, c\right\rangle_{X}$ has a pole of order $m$ at $\lambda_{0}$ if and only if $(\lambda I-A)^{-1}$ has a pole of order $m$ at $\lambda_{0}$.

Proof of Theorem 2.3. For $A$ on a finite-dimensional space, $A$ is cyclic if and only if $A^{*}$ is cyclic. To see this consider their characteristic and minimal polynomials. Since $\varphi(z)=\lambda \psi(\lambda)$ with $z=\lambda^{-1}$, it follows that $\varphi(z)$ has no pole in the closed unit disc $\overline{\mathbb{D}}$ if and only if $\psi(\lambda)$ has no pole in $(\mathbb{C} \cup\{\infty\}) \backslash \mathbb{D}$. For a rational $\varphi=\varphi(z), \varphi \in \mathbb{H}^{\infty}$ if and only if $\varphi$ has no pole in $\overline{\mathbb{D}}$. From these observations and Theorems 2.1 and 2.4, the assertion of the theorem readily follows.

We should mention the following two pioneering theorems in operator model theory. For these and further developments, see Radjavi and Rosenthal $[12, \S 3.5]$ and Sz.-Nagy and Foias [16].

TheORem 2.5 (Rota [14]). Let $A$ be a bounded operator on a complex Hilbert space of dimension equal to the cardinal number $\alpha$. If $r(A)<1$, then $A$ is similar to a part of the backward shift $S^{*}$ of multiplicity $\alpha$.

Theorem 2.6 (Foiaş [4] and de Branges and Rovnyak [2]). Let $A$ be a bounded operator on a complex Hilbert space $X$ such that $\|A\| \leq 1$ and $A^{n} x \rightarrow 0$ for all $x \in X$, and $\alpha$ be the dimension of the closure of the range of $I-A^{*} A$. Then $A$ is a part of the backward shift $S^{*}$ of multiplicity $\alpha$.

3. Proof of the main theorem. To prove the main theorem, we need some theorems of Nikol'skil and Helton. The following theorem is taken from Nikol'skiı [10]. We include the proof for the sake of completeness.

TheOREM 3.1 (Nikol'skiı̌ [10, p. 37]). Every $S^{*}$-invariant closed proper subspace $K \subset \mathbb{H}^{2}$ (i.e. $K \neq \mathbb{H}^{2}$ ) has a vector $f \in K$ that is cyclic for $S^{*} \mid K$.

Proof. Since $K^{\perp}=\mathbb{H}^{2} \ominus K \neq\{0\}$ is closed and $S$-invariant, there exists an inner $\Theta$ (i.e. $\Theta \in \mathbb{H}^{\infty},|\Theta(z)|=1$ a.e. on $\mathbb{T}$ ) such that $K^{\perp}=\Theta \mathbb{H}^{2}$ by the celebrated Beurling Theorem (see e.g. [15, p. 376]). Thus $K=\left(\Theta \mathbb{H}^{2}\right)^{\perp}$. Set $f=S^{*} \Theta$. Then for any $h \in \mathbb{H}^{2},\left\langle S^{*} \Theta, \Theta h\right\rangle=\langle\Theta, S \Theta h\rangle=\langle\Theta, z \Theta h\rangle=$ $\langle 1, \bar{\Theta} \Theta z h\rangle=\langle 1, z h\rangle=0$. Thus $f \in K$.

Now suppose that for some $k \in K,\left\langle\left(S^{*} \mid K\right)^{n} f, k\right\rangle=\left\langle S^{* n+1} \Theta, k\right\rangle=0$ for all $n \geq 0$. Note that $z^{-(n+1)}=\bar{z}^{n+1} \in \mathbb{L}^{2}$. Since $\left\langle z^{-(n+1)}, \bar{\Theta} k\right\rangle=$ $\left\langle\bar{z}^{n+1}, \bar{\Theta} k\right\rangle=\left\langle\Theta, z^{n+1} k\right\rangle=\left\langle\Theta, S^{n+1} k\right\rangle=\left\langle S^{* n+1} \Theta, k\right\rangle=0$ for all $n \geq 0$ for this $k$, we have $\bar{\Theta} k \in \mathbb{H}^{2}$. However, since $\langle\Theta h, k\rangle=0$ for any $h \in \mathbb{H}^{2}$, substituting $h=\bar{\Theta} k$, we have $\langle\Theta \bar{\Theta} k, k\rangle=\langle k, k\rangle=0$. Therefore $k=0$. This means that $f$ is cyclic for $S^{*} \mid K$. 
To proceed to Lemma 3.2 , recall that

$$
\mathrm{BMO}=\left\{f \in \mathbb{L}^{1}: \sup _{I} \frac{1}{|I|} \int_{I}\left|f\left(e^{i \theta}\right)-f_{I}\right| d \theta<\infty, f_{I}=\frac{1}{|I|} \int_{I} f\left(e^{i \theta}\right) d \theta\right\} .
$$

For BMO, see e.g. [10, p. 376].

LEMma 3.2. For an $S^{*}$-invariant closed subspace $K \subseteq \mathbb{H}^{2}$, suppose that $f \in K$ is cyclic for $S^{*} \mid K$. If $f \in \mathbb{H}^{\infty}$, then $f$ is continuously cyclic for $S^{*} \mid K$.

Proof. Since $\mathbb{H}^{\infty} \subset \mathrm{BMO}$ as is easy to check, $f \in$ BMO. Let $f=$ $\sum_{n=0}^{\infty} f_{n} z^{n}$. Then the cyclicity operator $\mathcal{C}$ can be represented by the infinite Hankel matrix $\left(f_{m+n}\right)$ if one regards $\mathbb{H}^{2}$ as $l^{2}$ under the natural correspondence $\mathbb{H}^{2} \ni \sum_{n=0}^{\infty} h_{n} z^{n} \mapsto\left(h_{n}\right)_{n=0}^{\infty} \in l^{2}$. Note that this correspondence gives an isometrical isomorphism between $\mathbb{H}^{2}$ and $l^{2}$. The lemma follows from Nehari's theorem that $\left(f_{m+n}\right)$ is bounded if and only if $f \in \mathrm{BMO}$ (see e.g. $[10$, p. 376, p. 408]).

Throughout let $e_{0}=1 \in \mathbb{H}^{2}$. For a closed subspace $K \subseteq \mathbb{H}^{2}$, let $P_{K}$ denote the orthogonal projection of $\mathbb{H}^{2}$ onto $K$.

Lemma 3.3. For an $S^{*}$-invariant closed subspace $K \subseteq \mathbb{H}^{2}$, set $g=$ $P_{K} e_{0}$. Then $g$ is strongly cyclic for $\left(S^{*} \mid K\right)^{*}$.

Proof. Define the cyclicity operator $\mathcal{O}: l^{2} \rightarrow K$ for $g$ and $\left(S^{*} \mid K\right)^{*}$ by

$$
\mathcal{O} y=\sum_{n=0}^{\infty} y_{n}\left(S^{*} \mid K\right)^{* n} g
$$

for $y=\left(y_{n}\right)_{n=0}^{\infty} \in l^{2}$. We show that $\mathcal{O}$ is continuous and surjective.

For any $k \in K, \sum_{n=0}^{\infty}\left\langle k, S^{n} e_{0}\right\rangle S^{n} e_{0}=k$. Note that $S^{n} e_{0}=z^{n} \in \mathbb{H}^{2}$. Since $P_{K} k=k$, we have $\left\langle k, S^{n} e_{0}\right\rangle=\left\langle P_{K} k, S^{n} e_{0}\right\rangle=\left\langle k, P_{K} S^{n} e_{0}\right\rangle$ and so

$$
\sum_{n=0}^{\infty}\left\langle k, P_{K} S^{n} e_{0}\right\rangle P_{K} S^{n} e_{0}=k .
$$

Recall that $K$ is $S^{*}$-invariant and hence is $\left(S^{*} \mid K\right)^{*}$-invariant as well. So for any $h \in \mathbb{H}^{2}$ we have

$$
\begin{aligned}
\left\langle P_{K} S^{n} e_{0}, h\right\rangle & =\left\langle S^{n} e_{0}, P_{K} h\right\rangle=\left\langle e_{0}, S^{* n} P_{K} h\right\rangle=\left\langle e_{0}, P_{K}\left(S^{*} \mid K\right)^{n} P_{K} h\right\rangle \\
& =\left\langle P_{K} e_{0},\left(S^{*} \mid K\right)^{n} P_{K} h\right\rangle=\left\langle g,\left(S^{*} \mid K\right)^{n} P_{K} h\right\rangle \\
& =\left\langle\left(S^{*} \mid K\right)^{* n} g, P_{K} h\right\rangle=\left\langle P_{K}\left(S^{*} \mid K\right)^{* n} g, h\right\rangle=\left\langle\left(S^{*} \mid K\right)^{* n} g, h\right\rangle .
\end{aligned}
$$

Therefore we have

$$
\left(S^{*} \mid K\right)^{* n} g=P_{K} S^{n} e_{0} .
$$

Now take an arbitrary $y=\left(y_{n}\right)_{n=0}^{\infty} \in l^{2}$. Then $h=h(z)=\sum_{n=0}^{\infty} y_{n} z^{n} \in$ $\mathbb{H}^{2}$. Recall that this correspondence gives the natural isometrical isomor- 
phism between $l^{2}$ and $\mathbb{H}^{2}$. Recall also that $S^{n} e_{0}=z^{n} \in \mathbb{H}^{2}$. Since

$$
\sum_{n=0}^{\infty} y_{n} S^{n} e_{0}=\sum_{n=0}^{\infty} y_{n} z^{n}=h,
$$

we have

$$
\sum_{n=0}^{\infty} y_{n}\left(S^{*} \mid K\right)^{* n} g=\sum_{n=0}^{\infty} y_{n} P_{K} S^{n} e_{0}=P_{K} h \in K .
$$

From this we see that $\mathcal{O}$ is continuous and surjective. Therefore by definition, $g$ is strongly cyclic for $\left(S^{*} \mid K\right)^{*}$.

Lemma 3.4. Let $K$ be an $S^{*}$-invariant closed subspace of $\mathbb{H}^{2}$. For $f \in K$ and $g=P_{K} e_{0}$, we have $\left\langle\left(I_{K}-z\left(S^{*} \mid K\right)\right)^{-1} f, g\right\rangle=f(z), z \in \mathbb{D}$. Here $I_{K}$ denotes the identity map on $K$.

Proof. Let $f(z)=\sum_{n=0}^{\infty} f_{n} z^{n}$. Since $S^{*} \mid K$ is a contraction, for $z \in \mathbb{D}$ we have

$$
\begin{aligned}
\left\langle\left(I_{K}-z\left(S^{*} \mid K\right)\right)^{-1} f, g\right\rangle & =\left\langle\sum_{n=0}^{\infty}\left(z S^{*} \mid K\right)^{n} f, P_{K} e_{0}\right\rangle=\left\langle P_{K} \sum_{n=0}^{\infty}\left(z S^{*} \mid K\right)^{n} f, e_{0}\right\rangle \\
& =\left\langle\sum_{n=0}^{\infty}\left(z S^{*} \mid K\right)^{n} f, e_{0}\right\rangle=\sum_{n=0}^{\infty}\left\langle\left(S^{*} \mid K\right)^{n} f, e_{0}\right\rangle z^{n} \\
& =\sum_{n=0}^{\infty} f_{n} z^{n}=f(z) .
\end{aligned}
$$

Here we view $\left(I_{K}-z\left(S^{*} \mid K\right)\right)^{-1} f=\sum_{n=0}^{\infty}\left(z S^{*} \mid K\right)^{n} f$ as an element of $K \subseteq$ $\mathbb{H}^{2}$ which depends on the parameter $z$.

To give the proof of the main theorem, we need the following theorem of Helton. Again for the sake of completeness, we give a detailed proof, focusing on the construction of the similarity operator.

Theorem 3.5 (Helton [7]). For $i=1,2$, let $X_{i}$ be a separable complex Hilbert space with an inner product $\langle\cdot, \cdot\rangle_{X_{i}}$. Let bounded operators $A_{i}: X_{i} \rightarrow$ $X_{i}$ and vectors $b_{i}, c_{i} \in X_{i}, i=1,2$, be such that $b_{i}$ is continuously cyclic for $A_{i}, c_{i}$ is strongly cyclic for $A_{i}^{*}$ and $\left\langle A_{1}^{n} b_{1}, c_{1}\right\rangle_{X_{1}}=\left\langle A_{2}^{n} b_{2}, c_{2}\right\rangle_{X_{2}}$ for all $n \geq 0$. Then $A_{2}$ is similar to $A_{1}$.

Proof. Throughout this proof let $i=1$ or 2 . Let $\mathcal{C}_{i} u=\sum_{n=0}^{\infty} u_{n} A_{i}^{n} b_{i}$ for $u=\left(u_{n}\right)_{n=0}^{\infty} \in l^{2}$ and $\mathcal{O}_{i} y=\sum_{n=0}^{\infty} y_{n} A_{i}^{* n} c_{i}$ for $y=\left(y_{n}\right)_{n=0}^{\infty} \in l^{2}$. Note that $\mathcal{C}_{i}$ and $\mathcal{O}_{i}$ are continuous by assumption. Thus $\mathcal{O}_{i}^{*}: X_{i} \rightarrow l^{2}$ is also continuous (see e.g. [3, p. 31]). It is easy to see that $\mathcal{O}_{i}^{*} x_{i}=\left(\left\langle A_{i}^{n} x_{i}, c_{i}\right\rangle_{X_{i}}\right)_{n=0}^{\infty}$ 
for $x_{i} \in X_{i}$. Thus it is easy to check that

$$
\mathcal{O}_{i}^{*} \mathcal{C}_{i} u=\left(\left\langle A_{i}^{n} \sum_{m=0}^{\infty} u_{m} A_{i}^{m} b_{i}, c_{i}\right\rangle_{X_{i}}\right)_{n=0}^{\infty}=\left(\sum_{m=0}^{\infty}\left\langle A_{i}^{n+m} b_{i}, c_{i}\right\rangle_{X_{i}} u_{m}\right)_{n=0}^{\infty} .
$$

Therefore $\mathcal{O}_{1}^{*} \mathcal{C}_{1}=\mathcal{O}_{2}^{*} \mathcal{C}_{2}$. Note that $\operatorname{im} \mathcal{O}_{1}^{*} \mathcal{C}_{1}=\operatorname{im} \mathcal{O}_{2}^{*} \mathcal{C}_{2} \subseteq \operatorname{im} \mathcal{O}_{1}^{*} \cap \operatorname{im} \mathcal{O}_{2}^{*}$ $\subseteq \operatorname{im} \mathcal{O}_{i}^{*}$.

Since $\mathcal{O}_{1}$ is a bounded surjection and $\operatorname{ker} \mathcal{O}_{1}^{*}=\left(\operatorname{im} \mathcal{O}_{1}\right)^{\perp}=\{0\}$, it follows that $\mathcal{O}_{1}^{*}: X_{1} \rightarrow l^{2}$ is a bounded injection. Note that im $\mathcal{O}_{1}^{*}=\left(\operatorname{ker} \mathcal{O}_{1}\right)^{\perp}$ and so $\operatorname{im} \mathcal{O}_{1}^{*}$ is closed. Therefore by the Inverse Mapping Theorem (see e.g. $\left[3\right.$, p. 94]), there exists a bounded bijection $\mathcal{O}_{1}^{* L}: \operatorname{im} \mathcal{O}_{1}^{*} \rightarrow X_{1}$ such that $\mathcal{O}_{1}^{* L} \mathcal{O}_{1}^{*}=I_{X_{1}}$. Thus $\mathcal{C}_{1}=\mathcal{O}_{1}^{* L} \mathcal{O}_{2}^{*} \mathcal{C}_{2}$. There exists a bijection $\mathcal{C}_{2}^{R}: \operatorname{im} \mathcal{C}_{2} \rightarrow$ $\left(\operatorname{ker} \mathcal{C}_{2}\right)^{\perp}$ such that $\mathcal{C}_{2} \mathcal{C}_{2}^{R}=I: \operatorname{im} \mathcal{C}_{2} \rightarrow \operatorname{im} \mathcal{C}_{2}$. Note that $\mathcal{C}_{2}^{R}$ need not be bounded. Since $\mathcal{O}_{2}^{*}: X_{2} \rightarrow \operatorname{im} \mathcal{O}_{2}^{*}$ and $\mathcal{O}_{1}^{* L}: \operatorname{im} \mathcal{O}_{1}^{*} \rightarrow X_{1}$ have bounded inverses, it follows that $\mathcal{C}_{1} \mathcal{C}_{2}^{R}=\mathcal{O}_{1}^{* L} \mathcal{O}_{2}^{*}: \operatorname{im} \mathcal{C}_{2} \rightarrow \mathcal{O}_{1}^{* L} \mathcal{O}_{2}^{*}\left(\operatorname{im} \mathcal{C}_{2}\right)$ is bounded and has a bounded inverse.

Since $b_{1}$ is cyclic for $A_{1}$, for every $x_{1} \in X_{1}$ there exists a sequence $u^{(n)} \in$ $l^{2}$ such that $\operatorname{im} \mathcal{C}_{1} \ni x_{1}^{(n)}=\mathcal{C}_{1} u^{(n)} \rightarrow x_{1}$. Since $\mathcal{O}_{1}^{*} \mathcal{C}_{1}=\mathcal{O}_{2}^{*} \mathcal{C}_{2}$, by setting $x_{2}^{(n)}=\mathcal{C}_{2} u^{(n)} \in \operatorname{im} \mathcal{C}_{2}$ we have $\mathcal{O}_{1}^{*} x_{1}^{(n)}=\mathcal{O}_{2}^{*} x_{2}^{(n)}$. Thus $x_{1}^{(n)}=\mathcal{O}_{1}^{* L} \mathcal{O}_{2}^{*} x_{2}^{(n)}$. This means that $\mathcal{O}_{1}^{* L} \mathcal{O}_{2}^{*}\left(\operatorname{im} \mathcal{C}_{2}\right)$ is dense in $X_{1}$. Of course $\operatorname{im} \mathcal{C}_{2}$ is also dense in $X_{2}$ by assumption. Therefore $T=\mathcal{C}_{1} \mathcal{C}_{2}^{R}=\mathcal{O}_{1}^{* L} \mathcal{O}_{2}^{*}$ extends to an invertible bounded bijection from $X_{2}$ to $X_{1}$ in the natural way.

It is also easily checked that $\mathcal{O}_{1}^{*} A_{1} \mathcal{C}_{1}=\mathcal{O}_{2}^{*} A_{2} \mathcal{C}_{2}$. Thus $A_{1} T=T A_{2}$ and so $A_{2}=T^{-1} A_{1} T$.

Now we can give the proof of the main theorem.

Proof of Theorem 2.1. (If) Set $f_{0}=\varphi \in \mathbb{H}^{\infty}$ and let $K=\overline{\operatorname{span}}\left\{S^{* n} f_{0}\right.$ : $n \geq 0\} \subseteq \mathbb{H}^{2}$. Here the closure is taken in the topology of $\mathbb{H}^{2}$. Obviously, $K$ is $S^{*}$-invariant and closed, and $f_{0}$ is cyclic for $S^{*} \mid K$. Moreover, by Lemma 3.2, $f_{0}$ is continuously cyclic for $S^{*} \mid K$.

Set $g=P_{K} e_{0}$. By Lemma 3.3, $g$ is strongly cyclic for $\left(S^{*} \mid K\right)^{*}$. By Lemma 3.4, $\left\langle\left(I_{K}-z\left(S^{*} \mid K\right)\right)^{-1} f_{0}, g\right\rangle=f_{0}(z)=\varphi(z)$. Thus all the conditions in Theorem 3.5 are satisfied for $A_{1}=S^{*} \mid K, b_{1}=f_{0} \in X_{1}, c_{1}=g \in X_{1}$ where $X_{1}=K$ and $A_{2}=A, b_{2}=b \in X_{2}, c_{2}=c \in X_{2}$ where $X_{2}=X$, and so $A$ can be recovered from $S^{*} \mid K$ by $T: X \rightarrow K$ as constructed in the proof of Theorem 3.5.

(Only if) Let $T: X \rightarrow K$ be a bounded bijection such that $K \subseteq \mathbb{H}^{2}$ is $S^{*}$-invariant and closed, and $A=T^{-1}\left(S^{*} \mid K\right) T$.

If $K=\mathbb{H}^{2}$, then, for example, $f=f(z)=e^{1 /(z-2)} \in \mathbb{H}^{\infty}$ is cyclic for $S^{*}=S^{*} \mid \mathbb{H}^{2}$, since $f$ does not have a meromorphic pseudocontinuation into $(\mathbb{C} \cup\{\infty\}) \backslash \overline{\mathbb{D}}$. See $\left[10\right.$, pp. 30-32]. As $f \in \mathbb{H}^{\infty}$, it is continuously cyclic for $S^{*}$ by Lemma 3.2 . 
If $K \neq \mathbb{H}^{2}$, then we can use Theorem 3.1 and set $f=S^{*} \Theta$ as in the proof of that theorem. Since $\Theta \in \mathbb{H}^{\infty}$ and $f(z)=\left(S^{*} \Theta\right)(z)=(\Theta(z)-\Theta(0)) / z$, we see that $f \in \mathbb{H}^{\infty}$. Thus by Lemma 3.2, $f$ is continuously cyclic for $S^{*} \mid K$.

In both cases, set $g=P_{K} e_{0}$. By Lemma $3.3, g$ is strongly cyclic for $\left(S^{*} \mid K\right)^{*}$. Now let $b=T^{-1} f, c=T^{*} g$. Then it is clear that $b$ is continuously cyclic for $A$, and $c$ is strongly cyclic for $A^{*}$. By Lemma 3.4, we have

$$
\begin{aligned}
\left\langle(I-z A)^{-1} b, c\right\rangle_{X} & =\left\langle\left(I-z T^{-1}\left(S^{*} \mid K\right) T\right)^{-1} T^{-1} f, T^{*} g\right\rangle_{X} \\
& =\left\langle\left(I_{K}-z\left(S^{*} \mid K\right)\right)^{-1} f, g\right\rangle=f(z) \in \mathbb{H}^{\infty}
\end{aligned}
$$

Acknowledgements. I would like to thank Professor Jaroslav Zemánek for many helpful comments. I would also like to thank the referees for the detailed review.

\section{References}

[1] V. M. Adamjan and D. Z. Arov, A class of scattering operators and of characteristic operator-functions of contractions, Dokl. Akad. Nauk SSSR (Ser. Mat.) 160 (1965), 9-12 (in Russian); English transl.: Soviet Math. Dokl. 6 (1965), 1-5.

[2] L. de Branges and J. Rovnyak, Canonical models of quantum scattering theory, in: Perturbation Theory and its Applications in Quantum Mechanics, C. H. Wilcox (ed.), Wiley, New York, 1966, 295-392.

[3] J. B. Conway, A Course in Functional Analysis, Springer, New York, 1985.

[4] C. Foiaş, A remark on the universal model of G. C. Rota for contractions, Com. Acad. R. P. Române 13 (1963), 343-352 (in Romanian, with Russian and French summaries).

[5] C. K. Fong, E. Nordgren, H. Radjavi and P. Rosenthal, Weak resolvents of linear operators, II, Indiana Univ. Math. J. 39 (1990), 67-83.

[6] P. A. Fuhrmann, Linear Systems and Operators in Hilbert Space, McGraw-Hill, New York, 1981.

[7] J. W. Helton, Discrete time systems, operator models, and scattering theory, J. Funct. Anal. 16 (1974), 15-38.

[8] P. D. Lax and R. S. Phillips, Scattering Theory, rev. ed., Academic Press, New York, 1989.

[9] W. Mlak, On a theorem of Lebow, Ann. Polon. Math. 35 (1977), 107-109.

[10] N. K. Nikol'skiǔ, Treatise on the Shift Operator: Spectral Function Theory, Springer, Berlin, 1986.

[11] E. Nordgren, H. Radjavi and P. Rosenthal, Weak resolvents of linear operators, Indiana Univ. Math. J. 36 (1987), 913-934.

[12] H. Radjavi and P. Rosenthal, Invariant Subspaces, Springer, Berlin, 1973.

[13] P. Rosenthal, Equivalents of the invariant subspace problem, in: Paul HalmosCelebrating 50 Years of Mathematics, J. H. Ewing and F. W. Gehring (eds.), Springer, New York, 1991, 179-188.

[14] G.-C. Rota, On models for linear operators, Comm. Pure Appl. Math. 13 (1960), 469-472.

[15] W. Rudin, Real and Complex Analysis, 2nd ed., McGraw-Hill, New York, 1974. 
[16] B. Sz.-Nagy and C. Foiaş, Harmonic Analysis of Operators on Hilbert Space, Akadémiai Kiadó, Budapest, and North-Holland, Amsterdam, 1970.

[17] Y. Uetake, On a property of weak resolvents and its application to a spectral problem, Ann. Polon. Math. 66 (1997), 263-268.

[18] - On a weak resolvent representation of a scattering matrix, Report No. 097/1999, Fac. of Math. and Comput. Sci., Adam Mickiewicz Univ., 1999.

Faculty of Mathematics and Computer Science

Adam Mickiewicz University

Matejki 48/49

60-769 Poznań, Poland

E-mail: uetake@amu.edu.pl

Received April 26, 2000

Revised version November 3, 2000 